\title{
Audit on documentation completeness after regional nerve blocks at University of Gondar referral hospital, 2018
}

\begin{abstract}
Documentation after regional nerve block is an essential component of patient pain management. It will safeguard against medico legal issues, it will increase the successful performance of regional anaesthesia, to safely follow patients having nerve block, to improve patient's recall of risks and benefits, to get briefed information during hand over of pain management and to request written rather than verbal communication during hand over of pain management. However, there are no specified protocols which should be recorded for each individual nerve block, less adequate documentation form and there is interpersonal variation during documentation here in this setup. So, we aimed to determine whether regional nerve block documentation trends in University of Gondar referral hospital meet the standards or not.

Methods: A cross sectional study is conducted from February 1-May 30, 2018.All patients who were given regional nerve block for postoperative pain management in the study period were included. A checklist used for data collection is prepared based on NYSORA Guideline recommendations.

Results: Forty-four record sheets were audited after patient's exposure of regional nerve block. The aseptic technique was documented for all patients. The level of documentation was $>85 \%$ for name of the nerve block and the availability of standard monitoring. However, the level of completeness of documentation was done below $50 \%$ for signature of service provider, consent, attempts of the technique, length of the procedure, approach of the nerve block, size of the needle, site of the block, time out period and level of sedation and indication of the nerve block. Conclusion and recommendation: The level of documentation after regional nerve block was unsatisfactory in our hospital compared with the recommendations of NYSORA guideline. So training should be given for all anaesthetists who will be involved in regional nerve block and regular re auditing should be done to attain the given standards.
\end{abstract}

Keywords: documentation, regional nerve block, pain management, clinical audit
Volume 10 Issue $6-2018$

\author{
Wubie Birlie Chekol,' Zewditu Abdissa \\ Denu, ${ }^{2}$ HailuYimer Tawuye, ${ }^{2}$ Abatneh Feleke \\ 'Lecturer, Department of Anesthesia, University of Gondar, \\ Ethiopia \\ ${ }^{2}$ Assistant professor, Department of Anesthesia, University of \\ Gondar, Ethiopia
}

Correspondence: Wubie Birlie Chekol, Lecturer, Department of Anesthesia, University of Gondar, Ethiopia, Te +251918200770, Email birlievubie@gmail.com

Received: November 12, 2018 | Published: November 15, 2017

\section{Introduction}

A nerve block is the injection of local anaesthetic agent near to the specific nerves to decrease pain in a certain part of patient's body during and after surgery. ${ }^{1,2}$ Regional nerve blocks are increasingly used as a component of multimodal analgesia and may be administered as single injection, catheter placement techniques or nerve stimulator technique. ${ }^{3,4}$ One excellent means of postoperative pain management with nerve block is that recording its conduct in the chart in a location separate from the anaesthetic record sheet. ${ }^{1,2,5}$ However, lack of adequate perioperative documentation has legal implications and can potentially affect the quality and safety of patient care. ${ }^{6}$ The importance of maintaining an adequate, accurate and legible documentation has been emphasized by different guidelines such as American Society of Anaesthesiologists (ASA) guidelines and New York Society of Regional Anaesthesiologists (NYSORA) guidelines

According to ASA guidelines for post-operative pain management documentation in conjunction with anaesthesia enable a provider to check for a regional anaesthetic technique separate from that of surgical anaesthesia if it is implemented before induction of anaesthesia or after anaesthesia emergence with primarily aim of postoperative pain management. The NYSORA guideline recommended that documentation after the nerve block placement should incorporates name of block performed, approach used, patient condition, indications for nerve block, patient position, needle design, technique, depth of insertion, local anaesthetic used, dose, standard monitoring, oxygen sources, suction machine, airway and emergency equipment availability. Documentation of the regional nerve block will safeguard against medico legal issues, it will increase the successful performance of regional anaesthesia, to safely follow patients having nerve block, to improve patients recall of risks and benefits, to get briefed information during hand over of pain management and to request written rather than verbal communication during hand over of pain management..$^{5,7-10}$

Practices of regional nerve block here in Gondar University Referral Hospital are expanding with multiple techniques and different approaches with increasing availability of equipments and medications. The demands of documentation of regional nerve block are growing well ,however there are no specified protocols which should be recorded for each individual nerve block, no separate record 
sheet availability, less adequate documentation form and there is interpersonal variation during documentation after the block. So this audit will help to enable anaesthetists' in practicing the best evidence based regional nerve block documentation techniques, to guide the practitioner to meet a given standard of care, to remind clinicians of basic minimum requirements for safety and quality of care for patients receiving a regional nerve block and to identify the gaps in current practice of regional nerve block documentation compared with the standards.

\section{Methods}

Ethical approval was obtained from Ethical Review committee, College of Medicine and Health Sciences, University of Gondar. A cross sectional study was conducted from February 1-May 30, 2018. All record sheets of patients who were exposed for regional nerve block during the study period were included in this clinical audit. A checklist that is developed from NYSORA guideline recommendations was used as data collection tool. Patient charts were used as a source of review after operation (Table 1).

Table I Data collection tools based on the recommendations of NYSORA guideline

\begin{tabular}{|c|c|c|c|}
\hline Standards & Target & Evidence & Data source \\
\hline Patient's identity & $100 \%$ & NYSORA & Chart \\
\hline Indication of nerve block & $100 \%$ & NYSORA & Chart \\
\hline Allergies and anticoagulation history & $100 \%$ & NYSORA & Chart \\
\hline Level of sedation during block & $100 \%$ & NYSORA & Chart \\
\hline The site of the nerve block & $100 \%$ & NYSORA & Chart \\
\hline Availability of Resuscitation equipments, oxygen sources & $100 \%$ & NYSORA & Chart \\
\hline Aseptic technique & $100 \%$ & NYSORA & Chart \\
\hline Time out period before needle insertion & $100 \%$ & NYSORA & Chart \\
\hline Time and date of nerve block & $100 \%$ & NYSORA & Chart \\
\hline Consent for nerve block & $100 \%$ & NYSORA & Chart \\
\hline Techniques of nerve block & $100 \%$ & NYSORA & Chart \\
\hline Name of the nerve block. & $100 \%$ & NYSORA & Chart \\
\hline The availability of standard monitoring & $100 \%$ & NYSORA & Chart \\
\hline Size of the needle & $100 \%$ & NYSORA & Chart \\
\hline Local anaesthetic given (dose, volume,concentration) & $100 \%$ & NYSORA & Chart \\
\hline Approach of the nerve block & $100 \%$ & NYSORA & Chart \\
\hline Length of the procedure & $100 \%$ & NYSORA & Chart \\
\hline Attempts of nerve block & $100 \%$ & NYSORA & Chart \\
\hline Signature of the service provider & $100 \%$ & NYSORA & Chart \\
\hline
\end{tabular}




\section{Results}

Forty-four record sheets were audited after patient's exposure of regional nerve block. The aseptic technique was documented for all patients. The level of documentation was $>85 \%$ for name of the nerve block and the availability of standard monitoring. However the level of completeness of documentation was done below $50 \%$ for signature of service provider, consent, attempting of the technique, length of the procedure, approach of the nerve block, size of the needle, site of the block, time out period and level of sedation and indication of the nerve block (Table 2) (Table 3).

Table 2 Factors that were audited based on NYSORA guideline recommendations for regional nerve block documentation, University of Gondar referral hospital, February I-May 30, 2018, 2018(N=44)

\begin{tabular}{lll}
\hline Standards & Frequency(No.) & Percent (\%) \\
\hline
\end{tabular}

Documentation of the clear identity

Yes

34

22.73

No

63.63

Yes

Allergies and anticoagulation history

Yes

NO

during block

Patient level of sedation

4

NO

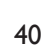

40

9.09

90.01

The site of block nerve block

Yes

14

31.81

No

30

68.18

Availability of resuscitation equipments and oxygen sources

Yes

34

77.27

No

10

22.73

Aseptic technique documentation

Yes

No

0

0

Time out period" before needle insertion.

Yes

No

0

44

100

The time of nerve block

Yes

No
4

40
Table 3 Factors that were audited based on NYSORA guideline recommendations for regional nerve block documentation, University of Gondar referral hospital, February I-May 30, 2018, 2018(N=44)

\begin{tabular}{lcc}
\hline Standards & Frequency (No.) & Per \\
\hline \multicolumn{2}{l}{ Consent for nerve block } \\
Yes & 0 & 0 \\
No & 44 & 100
\end{tabular}

The techniques of nerve block

$\begin{array}{lll}\text { Yes } \quad 16 & 36.36\end{array}$

No $\quad 28 \quad 63.64$

The name of nerve block

Yes $\quad 40 \quad 90.9$

NO $\quad 4.1$

Availability of standard monitoring

$\begin{array}{lll}\text { Yes } & 38 & 86.36\end{array}$

NO $\quad 6 \quad 13.64$

The size of needle

$\begin{array}{lll}\text { Yes } & 8 & 18.18\end{array}$

$\begin{array}{lll}\text { No } & 36 & 81.82\end{array}$

Local anaesthetic (dose, volume, concentration)

\begin{tabular}{|c|c|}
\hline Yes & 36 \\
\hline
\end{tabular}

$\begin{array}{lll}\text { No } & 8 & 18.19\end{array}$

Approach of the nerve block

$\begin{array}{lll}\text { Yes } & 8 & 18.18\end{array}$

$\begin{array}{lll}\text { No } & 36 & 81.81\end{array}$

Length of the procedure

$\begin{array}{lll}\text { Yes } & 6 & 13.63\end{array}$

$\begin{array}{lll}\text { No } & 38 & 86.37\end{array}$

Attempts of the nerve block

$\begin{array}{lll}\text { Yes } & 8 & 18.18\end{array}$

$\begin{array}{lll}\text { No } & 36 & 81.82\end{array}$

Signature of the service provider

$\begin{array}{lll}\text { Yes } & 0 & 0\end{array}$

$\begin{array}{lll}\text { No } & 44 & 100\end{array}$ 


\section{Discussion}

This audit determined whether regional nerve block documentation trends in University of Gondar referral hospital meet the standards or not. Complete regional nerve block documentation is vital for the quality of patient care. ${ }^{11}$ Only documentation of the aseptic technique gained $100 \%$ of this audit standard. On the other hand, signature of the service provider, consent of patient for regional nerve block and timeout period before needle insertion were not documented at all. Once regional nerve block is performed, it is vital to accurately document the procedure in detail by using a number of safety measures such as obtaining patient consent, ensuring appropriate monitoring, checking all equipments, drugs and performance of the correct surgical site. ${ }^{4}$ According to our clinical audit most of the factors that audited by the standard was below $50 \%$ such as signature of service provider, consent, attempting of the technique, length of the procedure, approach of the nerve block, size of the needle, site of the block, time out period, level of sedation and indication of the nerve block. The possible reason for inadequate documentation might be there are no specified protocols which should be recorded for each individual nerve block, less adequate documentation form, interpersonal variations, lack of reassessment of the chart after nerve block and inadequate training of practitioners.

According to literatures lack of adequate documentation after regional nerve block might have different adverse effects such as legal implications, high mortality and morbidity of patients, poor quality and safety of patient care. ${ }^{6}$ Also there might be a risk of nerve injury or other undesirable complications associated with the technique, so accurate documentation is important ${ }^{12}$ and helps meeting a legal requirements and as a source of education and audit. ${ }^{5}$ In general, full documentation after regional nerve block might enhance communication between the anaesthetist and surgical team in postoperative period. It also acts as a remainder to follow the appropriate steps in safely performing a regional nerve block. It reduces the potential complications inappropriate performances. ${ }^{4}$

\section{Conclusion and recommendation}

The level of documentation after regional nerve block was unsatisfactory in our hospital compared with the recommendations of the NYSORA guideline. There was no practice of documenting signature of the service provider, consent for nerve block and timeout period before needle insertion. So training should be given for all anaesthetists who will be involved in regional nerve block, preparing formal recording sheet for regional nerve block based on NYSORA guideline and regular re-auditing should be done to acquire the given standards.

\section{Acknowledgements}

We wish to submit an original audit entitled "Audit on documentation completeness after regional nerve blocks " for consideration by "Journal of anesthesia and critical care" we confirm that this work is original and has not been published elsewhere, nor is it currently under consideration publication elsewhere. We aimed to determine whether regional nerve block documentation trends at University of Gondar meet the standards or not.

\section{Conflict of interest}

Finally, we have no conflicts of interest to disclose.

\section{References}

1. Hadzic A, Vloka J, Kuroda M. Training requirements for peripheral nerve blocks. Curr Opin Anaesthesiol. 2002;(15):669-673.

2. Gonzalez KR, Murauski JD. Peripheral nerve blocks for postoperative analgesia. AORN Journal. 2002;1(7):136-154.

3. Joshi G, Gandhi K, Shah N, et al. Peripheral nerve blocks in the management of postoperative pain: challenges and opportunities. J Clin Anesth. 2016;(35):524-529.

4. Maran PJ, Fennessy P, Johonson MZ. Establishing a new national standard for the documentation of regional anesthesia in ireland. $B M J$. 2017;6(2):e000210.

5. Gerancher J, Viscusi E, Liguori G. Development of a Standardized Peripheral Nerve Block Procedure Note Form. Reg Anesth Pain Med. 2005;30(1):67-71.

6. Elhalawani I, Jenkins S. Perioperative anesthetic documentation:adherence to current australian guidlelines. $J$ Anaesthesiol Clin Pharmacol. 2013;29(2):211-215.

7. Bentin C, Rouge B. How to Report Post-op Pain Blocks. Outpatient surgery magazine. 2009:49-51.

8. Hardman D. Nerve injury after peripheral nerve block. Anesthesiology news. 2015:1-6.

9. Mariano E. Billing for Peripheral Nerve Blocks (United States).

10. Whiteley W, Foxall G, McCahon R, et al. Documentation of consent for peripheral nerve blockade. American Society of Regional Anesthesia and Pain Medicine. 2007;32(5):98.

11. Mathioudakis A, Rousalova I. How to keep good clinical records. Breathe (Sheff). 2016;12(4):369-373.

12. Hundson $\mathrm{P}$, Gnanaseakaran S. Improvement in documentation of epidural insertion with the addition of a sticker to the anesthetic chart:an audit cycle. Reg Anesth Pain Med. 2008;33(34). 